\title{
Exploiting the Tomographic Nature of the Ultrasound, Could Processed Ultrasound Images Be a Viable Alternative to Ct or MRI?
}

\author{
Mohammed Zahid Saadoon \\ Candidate for Fellowship of the Kurdistan Board for Medical Specialties, Maxillofacial \\ Surgery/Rizgary hospital, Erbil - Iraq \\ David I Adam \\ Employee at OSTEOOTECH UK LIMITED/London - UK \\ Jamal Abdul-Kader Al-Maki \\ University lecturer/ Collage of Medicine at Al-Iraqia University, Baghdad - Iraq \\ Reiadh Kamal Al-Kamali \\ Assistant Professor /College of Dentistry at Hawler Medical University, Erbil - Iraq
}

doi: $10.51505 / \mathrm{ijmshr} .2021 .5308$

URL: http://dx.doi.org/10.51505/ijmshr.2021.5308

\begin{abstract}
Introduction: Imaging is a very important procedure in many medical and engineering disciplines. It provides a non-destructive method of examination and exploring the interior anatomy and internal structure architecture of a certain domain. In medicine, different imaging modalities are available and many of them share the same concept, although having different processes to achieve it.

Aim of the study: to validate reconstruction of ultrasound images (derived from video format) could yield same results as in case of CT scan (directly constructed from DICOM data).

Materials and methods: A wooden model were used to obtain images using ultrasound and computerized tomography (CT), these images were processed to produce a 3 dimensional model. Results: initial results show that the capability of synthesis data from ultrasound imaging modality in a fashion similar to that performed routinely with CT scan data

Conclusion: ultrasound imaging is a very popular modality that could be used to compensate the shortcomings of other modalities

Keywords: ultrasound, CT-scan, Tomography, 3D reconstruction, DICOM

\section{Introduction}

Imaging is a very important procedure in many medical and engineering disciplines. $(1,2)$ It provides a non-destructive method of examination and exploring the interior anatomy and internal structure architecture of a certain domain. In medicine, different imaging modalities are available and many of them share the same concept, although having different processes to achieve it. (3)
\end{abstract}




\section{International Journal of Medical Science and Health Research}

Vol. 5, No. 03; 2021

ISSN: 2581-3366

Tissues are composite structures with complex isotropi city on different levels ( from gross anatomy down to the cellular level and further down to the molecular levels). (4) They have staged damage patterns that provide servo feedback for remodeling or repair(5). The different parts of tissues had different properties that could provide the ability to determine boundaries and detect the extension of the structures(6). All physical entities are measurable, but until the measuring tool is available it will remain unknown.

Many times measurement from different imaging modalities affects the decision of diagnosis, treatment, and follow-up. $(7,8)$

2D imaging such as plain X-ray and ultrasound is an indispensable tool in the medical armamentarium(9). Its usage could be expanded further to capture 3D data(10). There are many methods to achieve data acquisition. The ultrasound images could be reprocessed to get 3D data in many ways

- Using a specially designed ultrasound machine (11)

- Using a software algorithm to orient the captured data. This could be aided with the usage of a tracking device(12)

- Usage of mechanically controlled movement to determine the path of the probe(13). This type of movement provides more accurate data as it provides stable fixed precise movement, and any device could be implemented with it.

To achieve robust results mechanical control of the ultrasound probe could be regarded as more precise than the image processing by software, because it is not dependent upon the software algorithm, but rather on a precise movement of the mechanical stage. (14)

Ultrasound is already sectional (tomographic) radiographic modality(15). Theoretically, connecting the prop to the linear motorized stage will offer the same results for that in CT or MRI. 3D digital models could be obtained from the processing of successive images. (16)

We want to investigate the applicability of ultrasound to obtain 3D models and compare them to the standard benchmark modality that is used in both medical and engineering applications, which is the CT scan.

The ultrasound had many advantages:

- Suitable for both hard and soft tissues. It could give a clear idea about hard tissues contour $(17,18)$

- As the source is the same receiver, it allows a wide range of tissues manipulation and setting(19)

- a relatively small device for a wide range of application(19)

- Real-time data help for instant observation and modification of setting during an examination $(20)$ 


\section{International Journal of Medical Science and Health Research}

Vol. 5, No. 03; 2021

ISSN: 2581-3366

- The affordability allows a possible multi-probe arrangement(21). This would allow for a greater and more comprehensive region of interest. Wide page technology is an example of a good technique that is used in printing(22). This concept could be applied in ultrasound by using multiple probes to give wider ROI

- The widely available machine allows for a great room for development(23)

- No ionizing radiation is used that exposes the patient to its risk

- The fact that the ferromagnetic object is affected by the MRI preclude its usage where non-compatible cardiac pacemaker or where the implant is embedded in soft tissues such as aneurismal clips

But certain shortcomings must be noted when speaking about ultrasound implementation :

- resulted image quality dependent upon the spatial orientation of the probe, although artificial intelligence implementation could refine the results greatly(24)

- The prerequisite for ultrasound transmission through tissues and its reflection had a high limitation on the acquisition of the image. The ultrasound probe should be in close proximity to the examined part and a transmission medium must be in the interface

- Limitation in accessing deep tissue. have the more traveling of waves will result in more degradation of their energy and subsequently the image quality

- Nature of ultrasound waves results on the simultaneous effect of the resulted image that would even preclude Sciences of interpretational data (like mirroring, shadowing, contrast-enhancing. etc.)(25)

Our aim of this research is demonstrating whether the ultrasound imaging modality could produce a data that is substitute for other current conventional tomographic modalities

\section{Materials and methods}

Portable ultrasound system eZono ${ }^{\mathrm{TM}} 3000$ was used to obtain ultrasound imaging and SOMATOM Definition AS CT machine used to image the models.

The mechanical stage was custom made with a DC motor with a dial indicator holder was used to connect the ultrasound probe to the mobile part of the stage

We choose a hard object, wooden toys, provided in a local store and fix them on wooden plate, immersed all in the water in a plastic container.

A specific gel that is used in the hospitals could be used, but air entrapment could result in errors of reading. We had to choose a water path to provide waves transmission medium.

3 water resources had been chosen and tested, and their clarity was in the following arrangement:

- tap water

- water from bottle

- water from the home filtering system 
International Journal of Medical Science and Health Research

Vol. 5, No. 03; 2021

ISSN: $2581-3366$

Adobe Premiere had been used to process and trim the data provided from the ultrasound device. Avizo.v9.0.1software was used to analyze and process both datasets, from CT and ultrasound modalities. 3D models were further processed and aligned in Autodesk Netfabb for sake of comparison. The software was installed on industrial workstation e Pro 500g6.

\section{Results}

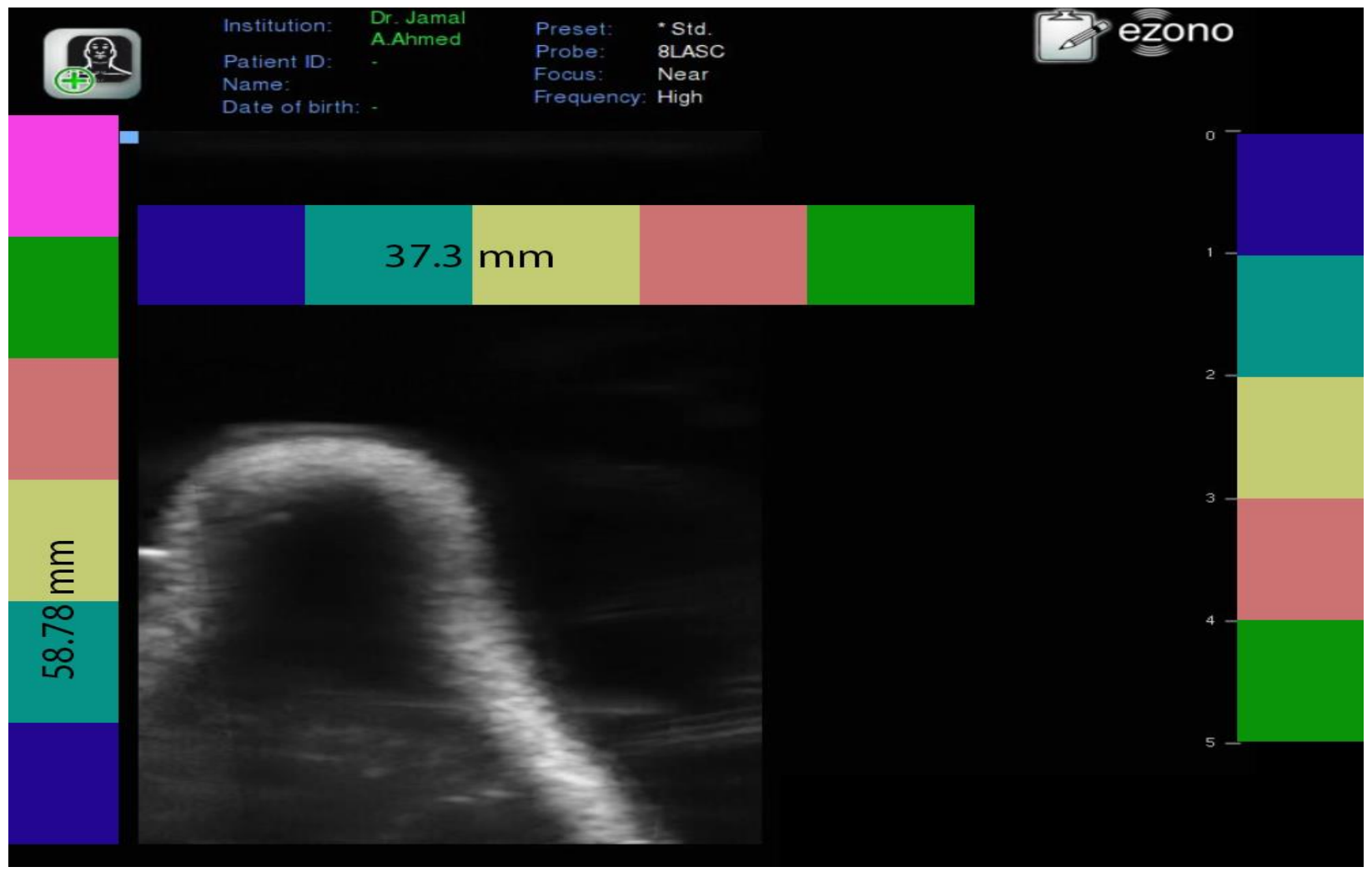

The frame of the ultrasound picture is $37.3 \mathrm{~mm} * 58.78 \mathrm{~mm}$, and this view is analog to the axial view of the CT scan modality. We have two sets of data that resulted from both modalities

- 2D data from the main plane of each modality. Axial in case of CT and the plane of the probe in case of ultrasound data. We can synthesis 2D images in the other planes in other orientations and directions.

In both modalities, the resultant 2D image in other planes are dependable on the resolution of the main plane, especially in our case the slice thickness of CT was $0.6 \mathrm{~mm}$ which is very fine and regarded acceptable.

The ultrasound had a higher frame rate (60 frames per second), and then the resultant 2D images in other planes in case of ultrasound would be much greater in resolution than that in resultant from CT. 


\section{International Journal of Medical Science and Health Research}

Vol. 5, No. 03; 2021

ISSN: 2581-3366

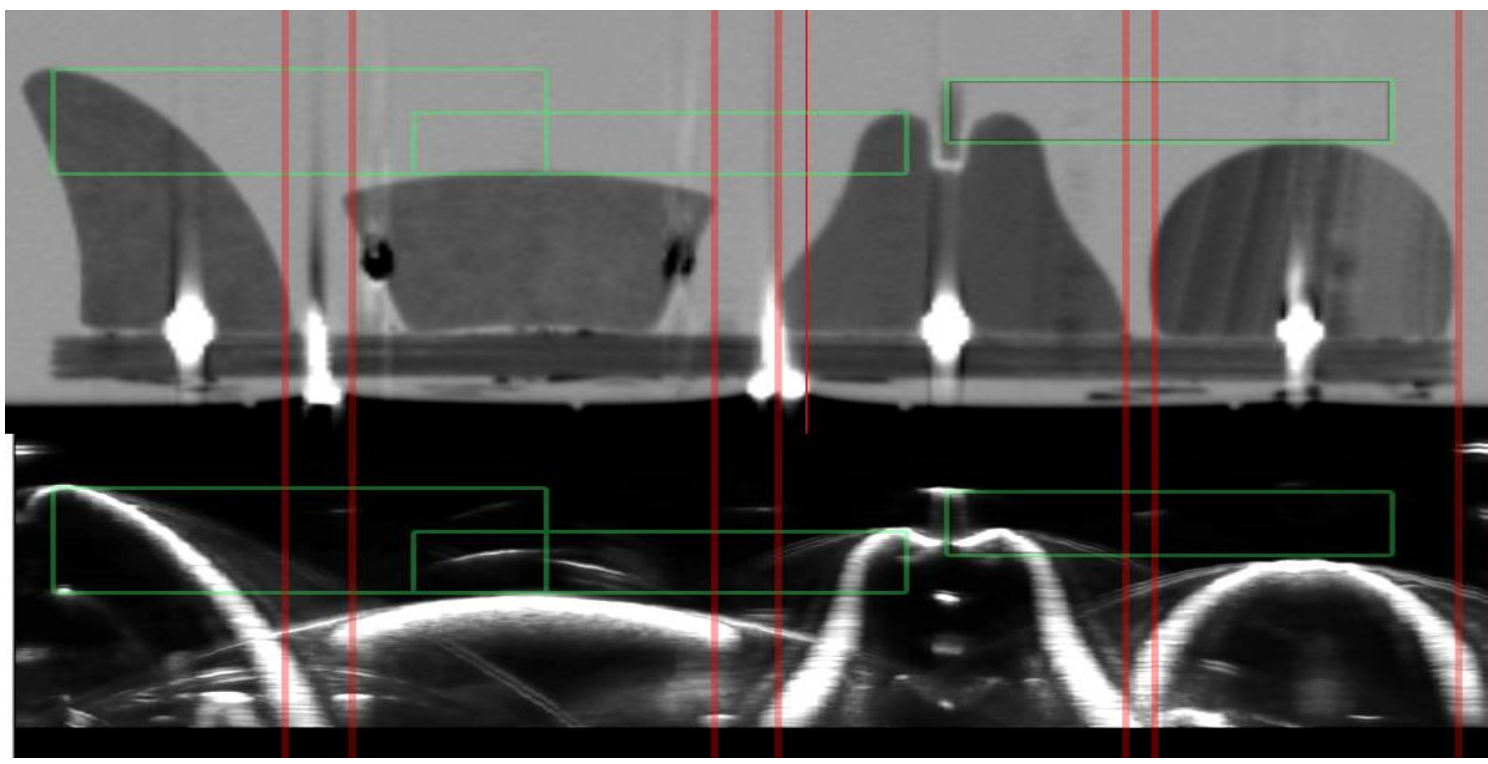

The upper picture represents the reprocessed axial image of the CT and what could be regarded as the sagittal reconstruction. The lower photo represents the same process for the data of the ultrasound. The Green markers in the upper photo had the same size and relative position as that in the lower photo. This indicates an approximation of both imaging modalities to each other

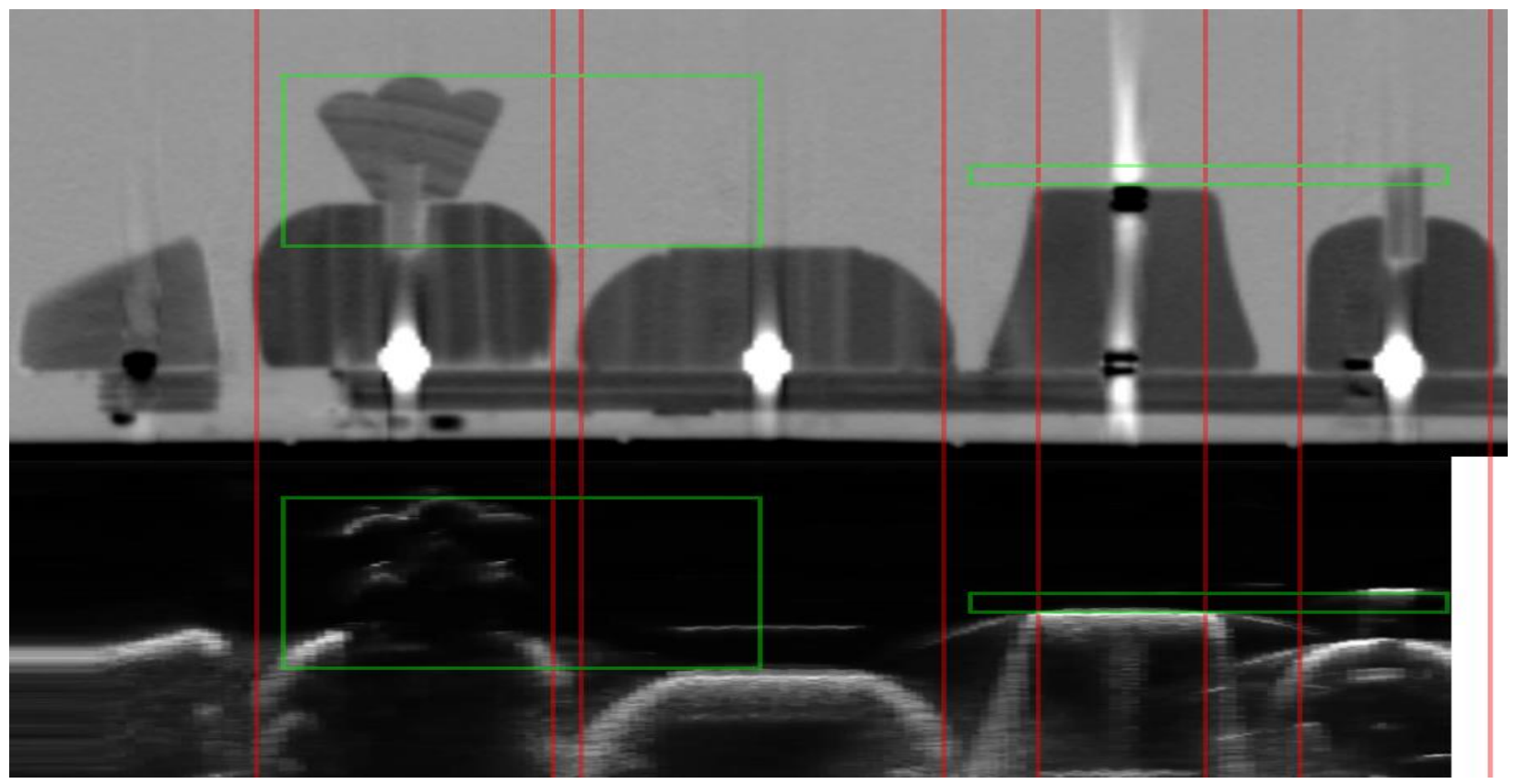

The effect of the shadow is apparent in the $2^{\text {nd }}$ sub model in the left-hand direction, where the crown-like shape obscures the pathway of the waves to capture the undercuts 


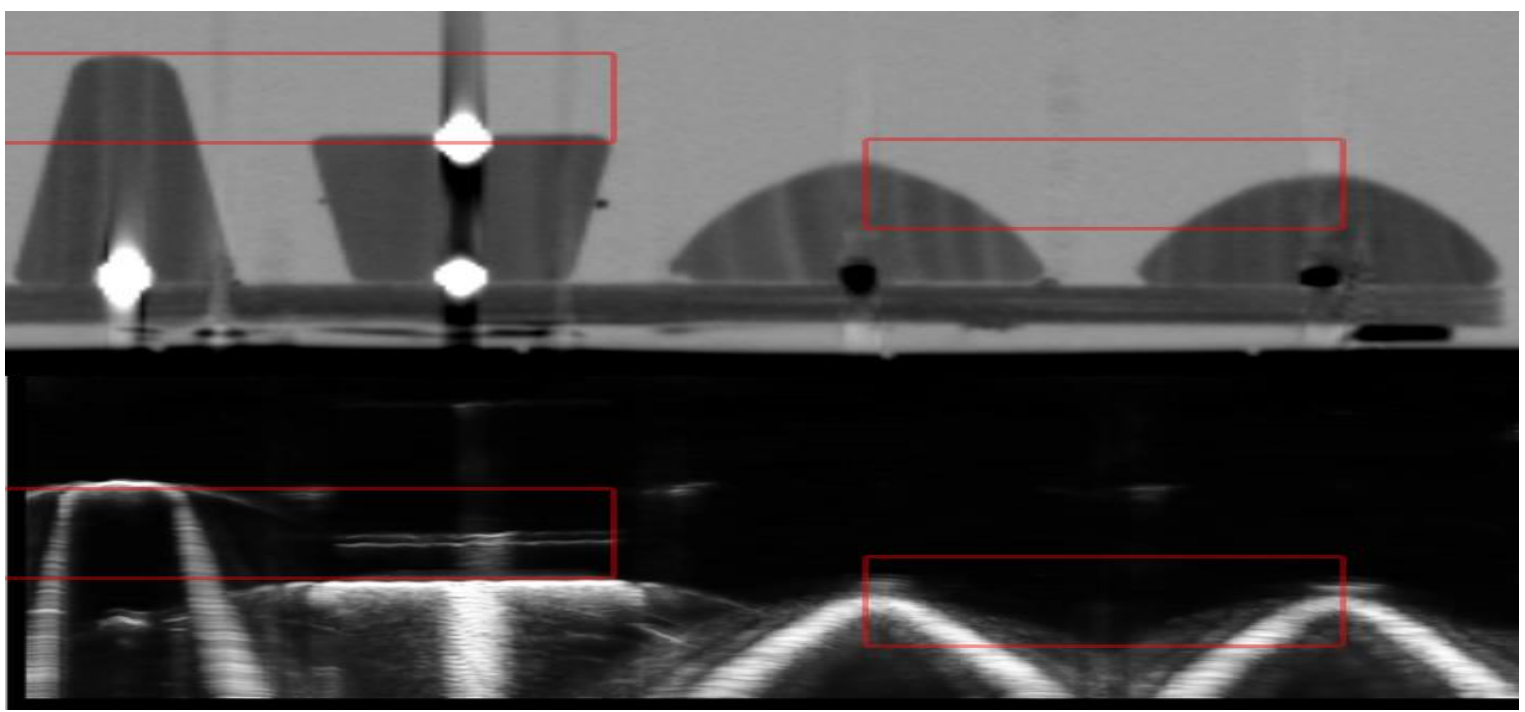

- 3D data in case of CT the greater contrast and consistency between successive images resulted in a much more smooth outer surface of their models in contrast to that 3D model resulted from ultrasound. We couldn't retrieve a model candidate for engineering inspection, as we previously did in case of craniofacial skeleton, so that rough estimation had been done which is could be regarded at least promising for further developments

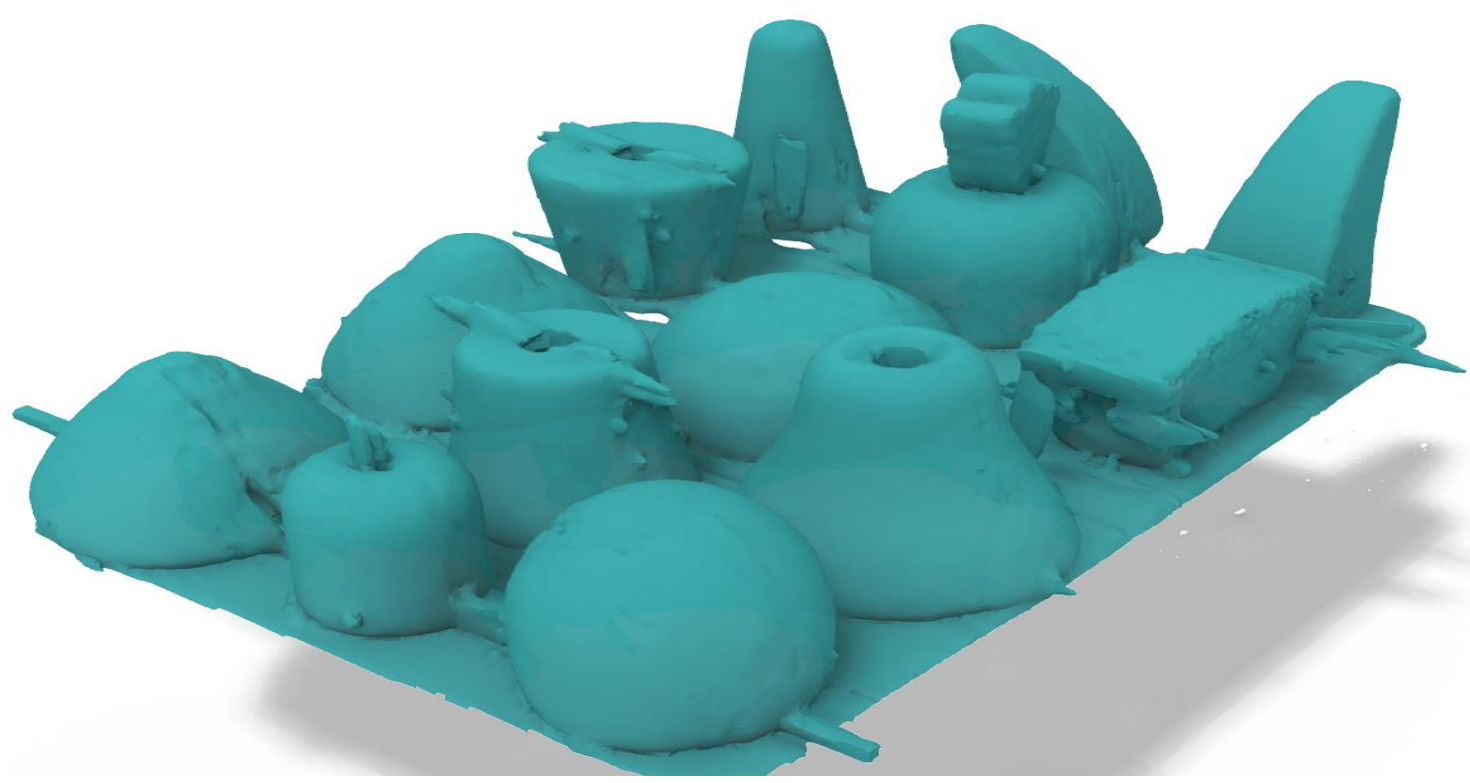

The 3D model retrieved from the CT data is very close to reality, the effect of the artifacts caused by the presence of the metallic objects (screw and the magnets) causes' apparent deformity close to the base 


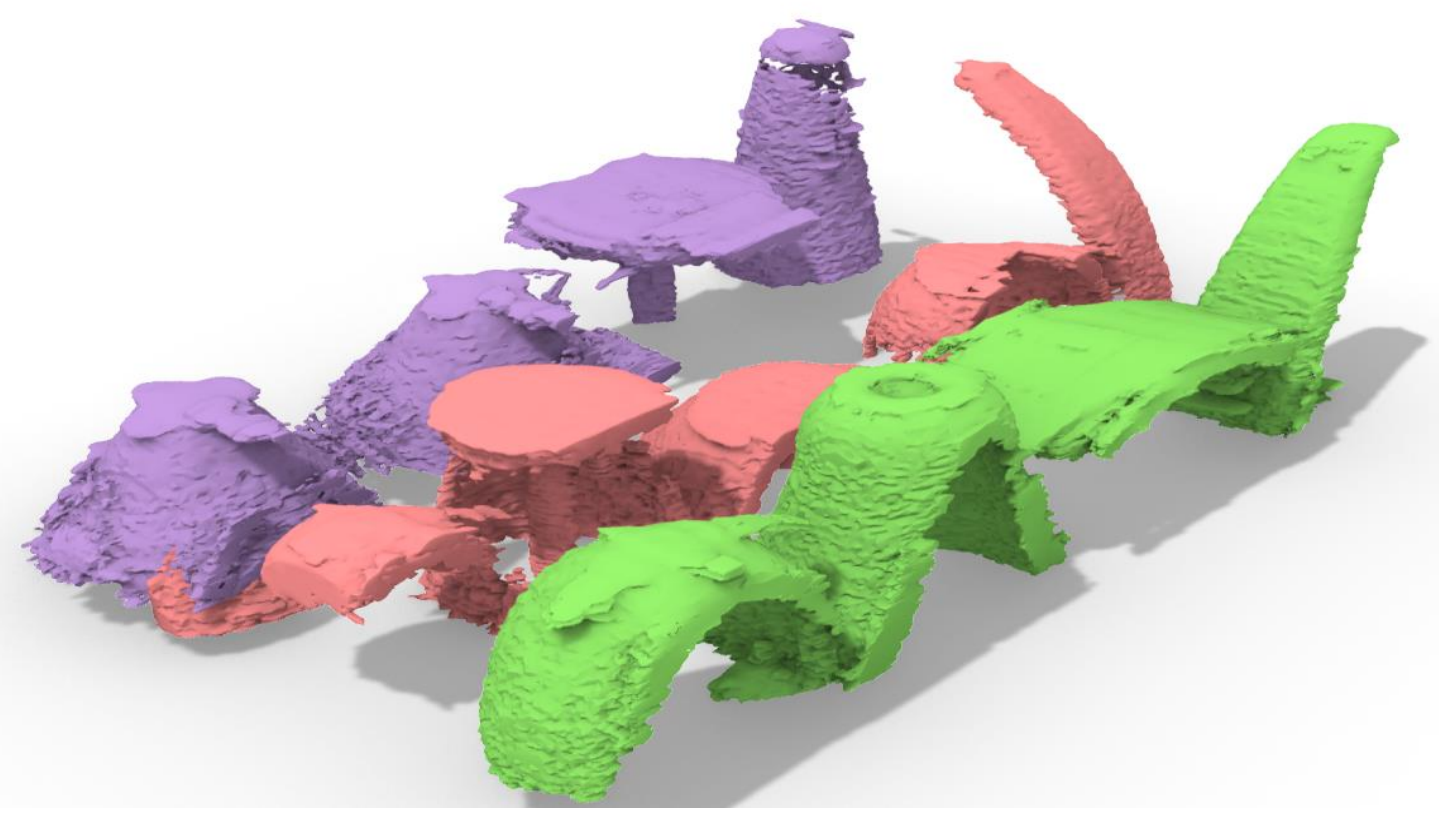

The 3D model retrieved from the ultrasound clearly depicting the inherited limitation of the ultrasound imaging modality. It has an irregular surface and each raw had been captured from separate reading in contrast to the $\mathrm{CT}$ where the whole model is captured at once in one session

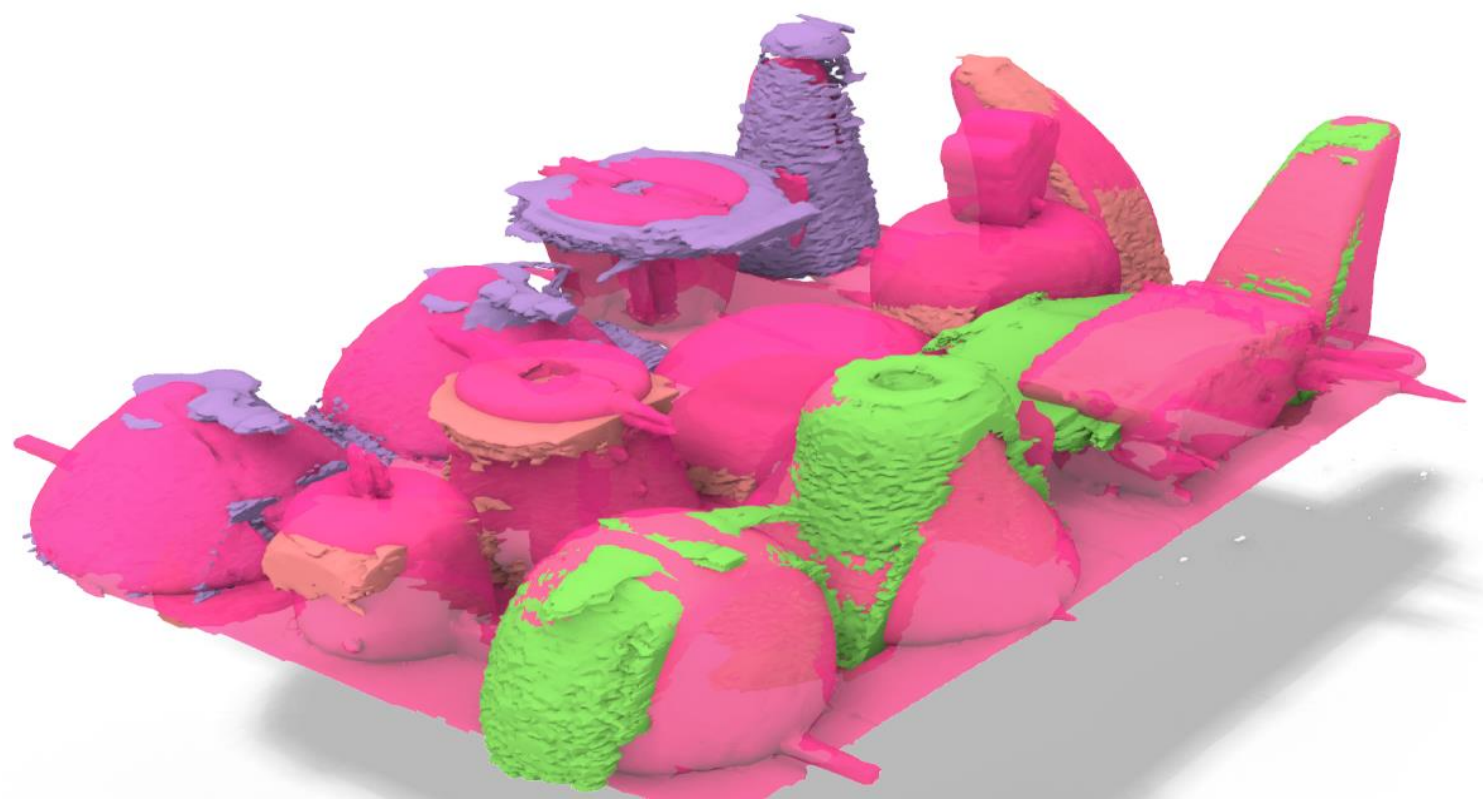

The superimposition of the 3D models on each other clearly show the difference but also show some sort of coincidence 


\section{International Journal of Medical Science and Health Research}

Vol. 5, No. 03; 2021

ISSN: 2581-3366

We also explored inclined oriented probe relative to the plane of the main axis of linear motion is perpendicular to this, which had resulted in distorted processed images in other planes

This distortion is well recognized when the gantry is tilted in the case of CT imaging modality (26) and should be considered when arranging the linear stage connection to the probe. The resultant images could be corrected using a specific formula, as in the case of CT image when the gantry is tilted.

The benefit of different angulation orientation of the probe is that the ultrasound image has resulted in different diagnosis or different results when the robe spatial orientation is changed. This is well known in the ultrasound.(27)

The resulting image of the ultrasound imaging modality could not be processed in a straightforward way as in the case of CT. Images processing that is taken by the CT machine, due to its nature, had enough contrast to yield a smooth 3D model.(28)

Nevertheless, the image processing recorded from the ultrasound device yield every important insight about the possible exploitation of the ultrasound imaging modality to reveal the complete picture of a certain anatomical region

The image quality with the curvilinear probe was not optimum to yield an analyzable model, also we think it was not a candidate to be processed to give images in the other planes. (29) Nevertheless in the future further trial is needed

We must emphasize the transmission medium that is used must be very clear. Tap water is full of what we thought it is microbubbles that are apparent in the ultrasound device. We think that degassing and filtering should be considered.

It must be noted here that tap water provides a very poor medium. We suspect that microbubbles had that effect so a degassed medium should be used to obtain a clearer image.

\section{Discussion}

To evaluate the accuracy of the processing of our sample in the case of ultrasound the submodels have been measured in the processed image in planes other than the main plane and compared to the CT reprocessed images.

The maximum diameter in the case of an ellipsoid or wall model and the dimensions of the irregular models.

This 2D comparison was done as the 3D model could not be retrieved from the ultrasound data. We also had the between each submodel and compared these measured to the in case of CT.

The phantom was made from softwood with relatively thick paint over it. This configuration could be one of the reasons that stand behind the degradation of resultant ultrasound image 


\section{International Journal of Medical Science and Health Research}

Vol. 5, No. 03; 2021

ISSN: $2581-3366$

quality in the surface region of the phantom. The resultant surface of the phantom units was irregular, which could be due to:

- limited capability of the used ultrasound unit

- Sub-optimal properties of the Phantom

Artificial intelligence (AI) application could be the approach to solve this problem of image degradation of the ultrasound modality.(30)

One of the limitations of ultrasound is the inability to capture undercuts in the case where a hard object (totally echogenic objects like bone or wood) is being imaged. This was evident with our ultrasound imaging result. The presence of a metallic head had to provide a transparent echogenic spot in the ultrasound modality, in contrast to the CT where it causes artifacts. (31)

Although we had good image processed in other planes that could be read by the human eye, the resultant 3D model gives an idea that they are coincident with the model resulted from CT. Our initial goal to retrieve a model with good surface quality that is applicable to be compared to the CT results (32) had not been attained. We will continue try to reach a such goal.

\section{Conclusion}

Ultrasound imaging modality is very powerful and further expansion of its application could cover the shortage of other imaging modalities

Possible further expansion of this experimental analysis for

- Evaluation of the pathological tissues of the soft tissues components as depth of tumor is very important to be defined

- Evaluation of mechanical properties of soft tissues

- Exploration of the different tissues 3D configuration

- Possible soft tissues 3d model synthesis

- Possibility of dynamic 3D evaluation of soft tissue, like heart and lung

- Bone surface evaluation is easily captured which would preclude the necessity to expose the patient to ionizing radiation as many cases as in the case of orthopedic surgery for pregnant women or in case of soft tissue that is more radiosensitive such as glands

- Possibility of application of the mechanical stage with this modality which results in more precise delivery of drug or tissue sampling

- It could be applied in forensic medicine as feasible effective technique

Thanks to Dr.Rawand Hawezi and the stuff of EMC hospital radiology department for generous cooperation in providing the CT examination to our phantom model.

\section{References}

1. Computer-assisted Orthopaedic Surgery. . Hernandez, D., Garimella, R., Eltorai, A., \& Daniels, A. H. (2017)., Orthopaedic surgery, 9(2), 152-158. https://doi.org/10.1111/os.12323. 


\section{International Journal of Medical Science and Health Research}

Vol. 5, No. 03; 2021

ISSN: 2581-3366

2. Application of microCT to the non-destructive testing of an additive manufactured titanium component. Blaine, Deborah \& Du Plessis, Anton \& Le Roux, Stephan \& Els, Johan \& Booysen, G.. s.l. : Case Studies in Nondestructive Testing and Evaluation. 4. 1-7., (2015).

3. Modalities for image- and molecular-guided cancer surgery. . Stammes MA, Bugby SL, Porta T, Pierzchalski K, Devling T, Otto C, Dijkstra J, Vahrmeijer AL, de Geus-Oei LF, Mieog JSD. s.l. : Br J Surg. , 2018 Jan.

4. 2011)., Rami K. Korhonen and Simo Saarakkala (November 25th. Biomechanics and Modeling of Skeletal Soft Tissues. [book auth.] Vaclav Klika. Theoretical Biomechanics. s.l. : IntechOpen.

5. Mechanical confinement regulates cartilage matrix formation by chondrocytes. . Lee HP, Gu L, Mooney DJ, Levenston ME, Chaudhuri O. s.l. : Nat Mater., 2017 Dec.

6. Using real-time ultrasound to teach living anatomy: an alternative model for large classes. Stringer MD, Duncan LJ, Samalia L. s.l. : N Z Med J., 2012 Sep 7.

7. Sports Injuries about the Hip: What the Radiologist Should Know. . Hegazi TM, Belair JA, McCarthy EJ, Roedl JB, Morrison WB. s.l. : Radiographics. , 2016 Oct.

8. Imaging and estimation of tissue elasticity by ultrasound. . BS.,. s.l. : Ultrasound Q., 2007 Dec.

9. How useful is muscle ultrasound in the diagnostic workup of neuromuscular diseases? van Alfen N, Gijsbertse K, de Korte CL. s.l. : Curr Opin Neurol. , 2018 Oct.

10. Freehand $3 D$ ultrasound imaging system using electromagnetic tracking. M. I. Daoud, A. Alshalalfah, F. Awwad and M. Al-Najar, ". s.l. : International Conference on Open Source Software Computing (OSSCOM), 2015.

11. Three-dimensional ultrasound imaging. . Fenster A, Downey DB, Cardinal HN. s.l. : Physics in Medicine and Biology, 2001.

12. Quaternion based freehand $3 D$ baby phantom reconstruction using $2 D$ ultrasound probe and game controller motion and positioning sensors. Mohamed F, Mong WS, Yusoff YA. s.l. : International Conference for Innovation in Biomedical Engineering and Life Sciences, 2015.

13. 3D freehand ultrasound reconstruction using a piecewise smooth Markov random field. Moon H, Ju G, Park S, Shin H. s.l. : Computer Vision and Image Understanding. , 2016.

14. An accurate and effective FMM-based approach for freehand $3 D$ ultrasound reconstruction. Wen T, Zhu Q, Qin W, Li L, Yang F, Xie Y, et al. s.l. : Biomedical Signal Processing and Control., 2013.

15. N.J. Watson. Ultrasound tomography. [book auth.] Mi Wang. Industrial Tomography. s.l. : Woodhead Publishing, 2015.

16. Micro-computed tomography assessment of different obturation techniques for filling lateral canals. Fragachán M, Pons M, Barriuso E, Frigola J, Ballester ML, Berástegui E. s.l. : J Clin Exp Dent. , 2018 Jul. 


\section{International Journal of Medical Science and Health Research}

Vol. 5, No. 03; 2021

ISSN: 2581-3366

17. Fast Automatic Bone Contour Extraction in Ultrasound Images. . Kowal, Jens \& Amstutz, Christoph \& Ioppolo, James \& Styner, Martin \& Nolte, Lutz-Peter. s.l. : IEEE TRANSACTIONS ON MEDICAL IMAGING. 1. , (2002).

18. Hacihaliloglu I. Ultrasound imaging and segmentation of bone surfaces: A review. . 2017, Hacihaliloglu I. Ultrasound imaging and segmentation of bone surfaces: A review. Technology (Singap World Sci). and doi:10.1142/S2339547817300049, . s.l. : Technology (Singap World Sci)., 2017.

19. Azhari,. Basics of Biomedical Ultrasound for Engineers. s.1. : Wiley-IEEE Press, 2010.

20. Hofer,. Ultrasound Teaching Manual: The Basics of Performing and Interpreting Ultrasound Scans. s.1. : thieme, 2013.

21. Morelli, Luca et al. Role of abdominal ultrasound for the surveillance follow-up of pancreatic cystic neoplasms: a cost-effective safe alternative to the routine use of magnetic resonance imaging. s.l. : World journal of gastroenterology, 2019.

22. Bhatt, Jay \& Deardurff, Larrie \& Jakubek, Vladimir \& Ruud, Cory \& McManus, Richard \& Vasudevan, Sundar. (2015). Pigmented Ink Technology to Enable HP PageWide XL Printer Capability. s.l. : NIP \& Digital Fabrication Conference. , 2015.

23. Sahlani L, Thompson L, Vira A, Panchal AR. Bedside ultrasound procedures: musculoskeletal and non-musculoskeletal. . s.l. : Eur J Trauma Emerg Surg., 2016 Apr.

24. Benacerraf BR, Minton KK, Benson CB, Bromley BS, Coley BD, Doubilet PM, Lee W, Maslak SH, Pellerito JS, Perez JJ, Savitsky E, Scarborough NA, Wax J, Abuhamad AZ. Proceedings: Beyond Ultrasound First Forum on Improving the Quality of Ultrasound Imaging in Obstetrics and Gynecology. s.l. : J Ultrasound Med., 2018 Jan.

25. Kremkau FW, Taylor KJ. Artifacts in ultrasound imaging. . s.l. : J Ultrasound Med. , 1986 Apr.

26. Nasada T, Hirayama S, Tanooka M, Fujita T, Sakai T. Evaluation of gantry tilt correction in multidetector-row CT: effect on multi-planar reconstruction images. s.l. : ]. Nihon Hoshasen Gijutsu Gakkai Zasshi, 2003 Jan.

27. Use of angulation in the detection of tendinitis with US. Lehtinen A, Bondestam S, Taavitsainen M. s.l. : Eur J Radiol. , 1994 Aug.

28. Reconstruction of freehand $3 D$ ultrasound based on kernel regression. . Chen, $\mathbf{X}$., Wen, T., Li, X. et al. s.l. : BioMed Eng OnLine, 2014.

29. Comparison of curvilinear and linear ultrasound imaging probes for measuring crosssectional area and linear dimensions. . Warner, Martin \& Cotton, A \& Stokes, Maria. s.l. : Journal of medical engineering \& technology., 2008.

30. A Survey of Deep-Learning Applications in Ultrasound: Artificial Intelligence-Powered Ultrasound for Improving Clinical Workflow. . Akkus Z, Cai J, Boonrod A, Zeinoddini A, Weston AD, Philbrick KA, Erickson BJ. s.l. : J Am Coll Radiol. , 2019 Sep.

31. Ultralydskanning til påvisning af fremmedlegemer i ekstremiteterne [Ultrasound in the detection of foreign bodies in the extremities]. IB., . s.l. : Ugeskr Laeger., 1991 Sep .

32. Comparison between the Anatomical Models of. Mohammed Zahid Saadoon, Zainab Zahid Saadoon. s.l. : International Journal of Science and Research, 2017. 